\section{Time-dependent recontamination rates of sterilised dental instruments}

\author{
C. S. Barker, ${ }^{1}$ V. Soro, ${ }^{2}$ D. Dymock, ${ }^{3}$ M. Fulford, ${ }^{4}$ J. R. Sandy ${ }^{5}$ \\ and A. J. Ireland ${ }^{6}$
}

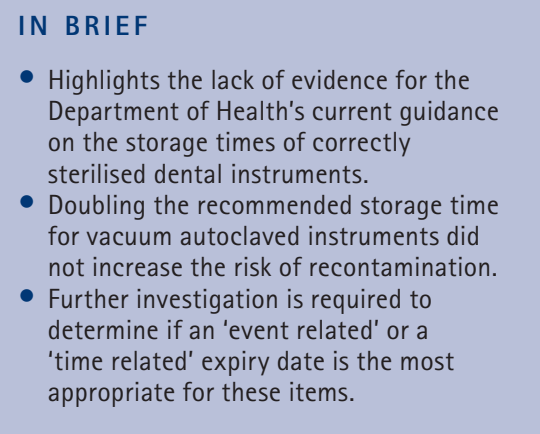

Objectives To determine time-related recontamination rates of sterilised instruments, following guidance from the UK Department of Health (HTM 01-05) that such instruments within primary dental care may only be stored for 60 days following sterilisation using a vacuum autoclave. Materials and methods A total of 25 used examination mirrors underwent a washer-disinfector cycle, individual packaging and finally vacuum autoclaving. Immediately after autoclaving, time zero, five mirrors were tested for microbial contamination by aerobic and anaerobic culture. At 31, 60, 90 and 124 days a further five mirrors were removed from their packaging and were similarly tested for microbial contamination. Results There was no bacterial growth on blood-enriched media under both aerobic and anaerobic conditions after 5 days of incubation at $37^{\circ} \mathrm{C}$ at any time period from 0 to 124 days post-sterilisation. Conclusions There was no recontamination of sterilised instruments in this investigation over the test period of 124 days. This exceeds the recommended limit of 60 days stated by the UK Department of Health. The new guidance, HTM 01-05, appears to place an extra burden on primary care dentists. This burden is not without associated costs, and at present does not appear to be based on published evidence.

\section{INTRODUCTION}

At present dental cross infection control protocols within the United Kingdom (UK) can vary according to setting. In particular they vary between primary care, in the community or high street, and secondary care in the hospital setting. These differences have traditionally been in the areas of decontamination before sterilisation, the method of sterilisation and the handling of the instruments post-sterilisation. Traditionally within the primary care setting instruments have been manually washed and then in some cases placed in an ultrasonic bath before sterilising in a downward displacement or vacuum autoclave.

\footnotetext{
"Specialist Registrar in Orthodontics, ${ }^{2}$ Research Technician in Microbiology, ${ }^{3}$ Senior Lecturer in Oral Microbiology, ${ }^{5}$ Professor and Honorary Consultant in Orthodontics, School of Oral and Dental Sciences, University of Bristol, Lower Maudlin Street, Bristol, BS1 2LY; ${ }^{4}$ Professional Lead for Dentistry, NHS Somerset, Wynford House, Lufton Way, Yeovil, Somerset, BA22 8HR; ${ }^{6}$ Consultant in Orthodontics, Royal United Hospitals, Bath/Reader in Orthodontics, School of Oral and Dental Sciences, Lower Maudlin Street, Bristol, BS1 2LY ${ }^{*}$ Correspondence to: Mr Christopher Barker Email: chris.barker@bristol.ac.uk
}

\section{Online article number E17}

Refereed Paper - accepted 21 April 2011

DOI: 10.1038/sj.bdj.2011.869

${ }^{\circledR}$ British Dental Journal 2011; 211: E17
Once sterilised they are either used directly or stored dry and wrapped. Within the secondary care setting the instruments have instead been placed in a washer disinfector before vacuum sterilisation in autoclavable sealed pouches, and again stored dry and wrapped. More recently an attempt has been made to improve the standard of decontamination within primary dental care following research in Scotland by the Glennie Group which highlighted the poor nature of decontamination in dental practices, ${ }^{1-3}$ trying to bring it closer to the standard expected in the secondary care setting. The new guidance to dentists in primary care on infection control is incorporated in the British Dental Association Advice sheet $A 12^{4}$ from advice given by the Department of Health document Health Technical Memorandum (HTM) 01-05: Decontamination in primary care dental practices, ${ }^{5}$ published in April 2009 and later revised in December 2009. This document is now available electronically online.

This guidance suggests that ultimately all primary care practices should use a validated washer-disinfector before sterilisation, something that has been mandatory within the hospital dental service for a number of years. The advice states that "wherever possible, cleaning should be undertaken using an automated and validated washer-disinfector in preference to manual cleaning; a washer-disinfector includes a disinfection stage that renders instruments safe for handling and inspection.' Although such decontamination and sterilisation procedures more closely approximate those seen in secondary care, there are still some differences, notably that in hospital central sterilisation facilities, the operatives are gowned to prevent contamination of clean instruments by personnel and that instead of clean and dirty zones within a sterilisation room, there are separate rooms used for clean and dirty instruments to reduce the risk of contamination.

HTM 01-05 recommends that reusable equipment must be thoroughly cleaned and sterilised (if appropriate) for reuse on other patients along the lines of the flow diagram in Figure 1. ${ }^{5}$ Instruments must also be sterilised before use if they are not purchased in a sterilised state. Critically, in the original HTM 01-05 guidance dated April 2009, bagged instruments that are then sterilised using a vacuum autoclave must be used within 30 days. This time limit 
was subsequently increased and amended to 60 days in December 2009 in the current version of HTM $01-05^{5}$ and yet there does not appear to be any published data to support the original or new time period.

\section{Instrument packaging and storage}

Following decontamination and before sterilisation in an autoclave, it is necessary to package instruments that are not to be used immediately. This packaging must protect against contamination that would render the instrument non-sterile, for example during transportation. However, there appears to be a dichotomy with respect to storage of sterilised instruments in the guidance issued by the Department of Health. HTM 01-05 (April 2009) ${ }^{5}$ states that sterilised instruments can only be stored for a period of 30 days in the case of Type B vacuum autoclaves and 21 days for Type N nonvacuum autoclaves. However, in the revised document HTM 01-05 December 2009, this figure for storage of instruments sterilised in a vacuum autoclave has increased to 60 days. At present there is no information in the literature on recontamination of sterilised dental instruments packaged in sealed pouches or the necessity to reprocess after a period of either 30 or 60 days. This expiry date also conflicts with guidance issued for the hospital service, Health Technical Memorandum (HTM) 2010, ${ }^{6}$ 2030, ${ }^{7}$ and 01-01: Decontamination of re-usable medical devices. ${ }^{8}$ Part five of the document HTM $2010^{6}$ acknowledges that time-related expiry dates have little value. It does not provide any specific guidance on the length of time a sterilised, packaged instrument can be stored, although it suggests the instruments should remain sterile for at least one year. The packaging itself should state 'sterile unless packaging opened or damaged'. The NHS produced a guide to decontamination of reusable surgical equipment in $2003^{9}$ which states that any sterilised instrument should be checked before use for any damage to the packaging; that appropriate sterilisation has occurred; and that the product is still within the expiry date. Again there is not a specified storage time except for "As a "rule of thumb", a product which has remained unused for more than six months should be deemed to be a product of over-stocking and an assessment undertaken as to its future need. ${ }^{9}$ Personal communication with

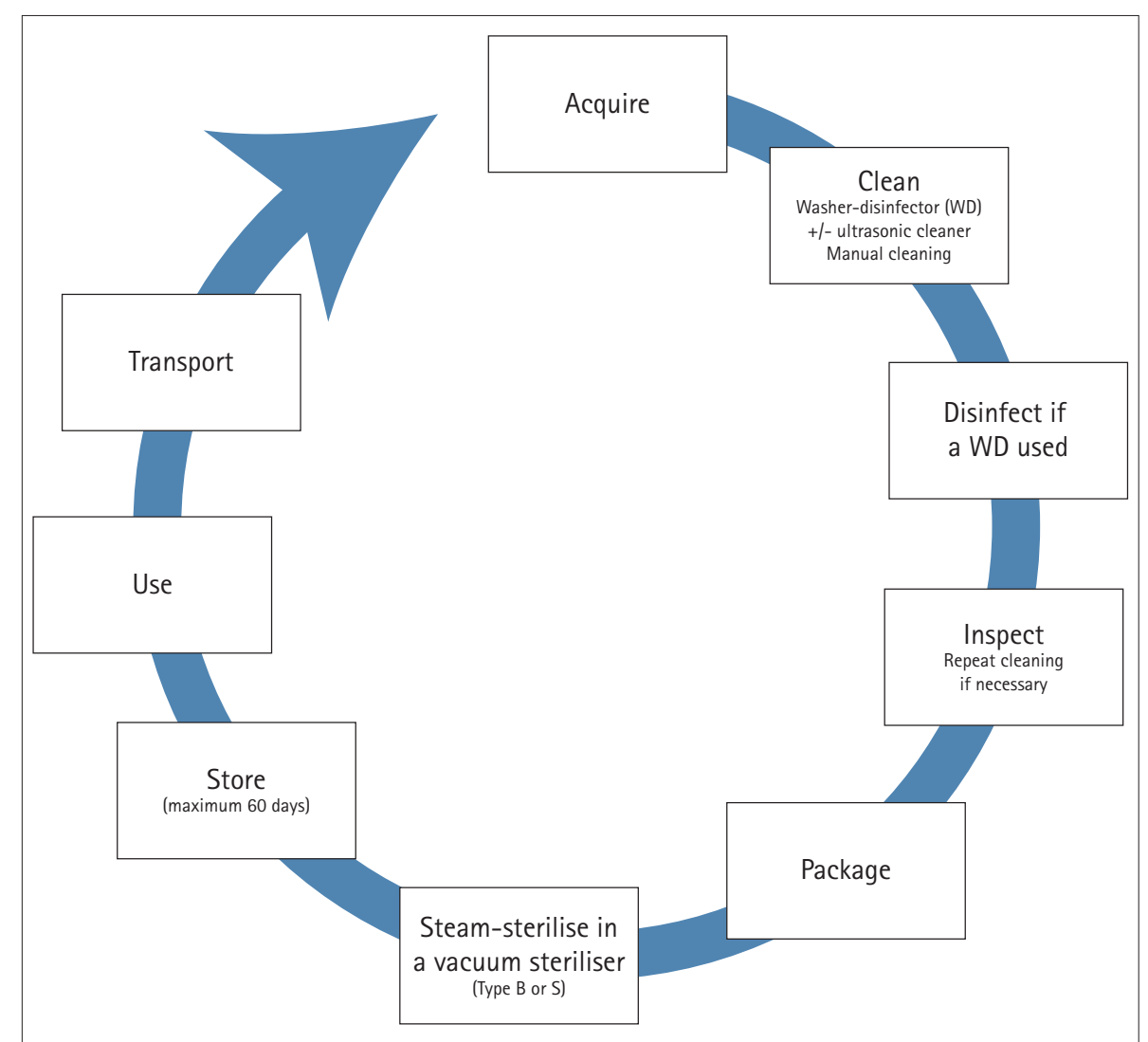

Fig. 1 The recommended primary care protocol for instruments and equipment (redrawn from HTM 01-05: decontamination in primary dental practices ${ }^{5}$ )

two local Sterile Service Departments has revealed they have differing policies regarding storage times of sterilised instruments. One NHS Trust advises that instruments can be stored for up to 12 months, or six months for theatre instruments, whereas another department advises a 24-month expiration date, as long as the packaging is still intact. Although this is only a small sample, it is clear that there is no consensus on storage time across NHS Trusts.

The Medicines and Healthcare Products Regulatory Agency (MHRA) also issue guidance on this subject, which is derived from the document Sterilisation, disinfection and cleaning of medical equipment: guidance on decontamination from the Microbiology Advisory Committee to Department of Health from 2006. ${ }^{10}$ In this document, which is soon to be superseded by the updated HTM 01-01, which was due in 2010, it states, 'The shelf-life of a packaged sterile instrument is event-related'. Event-related is defined as any event in which the sterilised package may become compromised leading to the possibility of contamination. The document continues, 'Provided the integrity of the packaging remains intact, that the packaging is not subject to any restrictions on use-life and it stays clean and dry, the instruments will remain sterile indefinitely'. This guidance appears to support storage times that are event-related rather than time-related. The United States (US) Centre for Disease Control \& Prevention (CDC), in its guidance to healthcare facilities published in 2008 (which includes dental offices), ${ }^{11}$ does discuss the evidence related to storage times and recontamination of sterile instruments. It concludes that sterility is event-related and not time-related, and recognises that a sterilised item should remain sterile until some event occurs which may lead to contamination. Many hospitals in the US have subsequently moved to the practice of event-related shelf life and in the US there is no disparity in the guidance issued to high street practices and hospitals.

Although there are some differences in the sterilisation protocols between primary dental care and hospital central sterilisation, namely differences in room design and greater aseptic conditions in central sterilisation departments, each undergoes disinfection before autoclave sterilisation. In dental practices, there is still continued 
use of the bench-top steriliser, which has been removed from central sterilisation departments. Type-B (vacuum) autoclaves are capable of validated sterilisation cycles and have been approved for use in primary care settings such as in general dental practice, general medical practice and podiatry by MHRA ${ }^{12}$ where central sterile service departments are not practical. Small steam sterilisers must conform to a European standard (EN13060) to be classed as suitable for medical, dental or veterinary use. The Medical Devices Agency provided guidance regarding the use of bench-top sterilisers in 2002, which stated, 'sterilised products will remain sterile indefinitely provided the packaging remains intact, clean and dry.' ${ }^{13}$ The lack of evidence for the guidance to primary care dentists has led to the research question: Is there any justification for the time related recontamination of sterilised instruments in UK dental practice?

\section{METHODS}

A total of 25 previously used dental examination mirrors (Ash ${ }^{\circledR}$ No.4 Plain mirror, Dentsply ${ }^{\circledR}$ ) were processed by the sterilisation service within Bristol Dental Hospital. The sterilisation service has a similar set-up to that in primary dental care, although there are separate rooms for decontamination of dirty instruments and sterilisation. The process comprises a washer-disinfector cycle (Belimed WD 230, Switzerland), individual packaging of each mirror in self-seal sterilisation pouches (BOP®DI (EN 868-5) Amcor Flexibles SPS, France) and autoclaving at $134^{\circ} \mathrm{C}$ for three minutes in a vacuum autoclave (MST-V Steam Steriliser, Belimed, Switzerland). Immediately after sterilisation, five mirrors were tested for microbial contamination to act as a baseline. This was undertaken by opening the individually packaged mirrors under laminar airflow (Bassaire) using aseptic techniques. Each item was swabbed using sterile woodstick swabs (Fisher), which were then placed in a $1 \mathrm{ml}$ buffer solution of 10:1 mMol Tris-EDTA. This solution was then vortexed using a Fisons Whirlimixer ${ }^{\mathrm{TM}}$ for 30 seconds to equally distribute the possible contaminates. A $100 \mu \mathrm{l}$ sample of the solution in each case was then plated onto bloodrich media, Blood Agar Base No. 2 and Fastidious Anaerobe Agar (LAB M Limited,
Bury) and then cultured i) aerobically, and ii) in an oxygen-depleted candle jar; a subset of samples (Day zero, 60, 90 and day 124) were also incubated in a Don Whitley Mark II Anaerobic Cabinet $\left(\mathrm{N}_{2}: \mathrm{H}_{2}: \mathrm{CO}_{2}\right.$ at $8: 1: 1)$ and all samples were incubated at $37^{\circ} \mathrm{C}$ for 5 days along with uncontaminated controls. The remaining mirrors were stored under the same conditions at airconditioned room temperature $\left(21^{\circ} \mathrm{C}\right)$, as would represent the normal clinical situation, until testing. Subsequent sets of five mirrors were tested in the same manner using the above protocol at 31, 60, 90 and 124 days.

\section{FINDINGS}

There was no bacterial growth on bloodenriched media in either aerobic and anaerobic conditions after five days of incubation at $37^{\circ} \mathrm{C}$ for mirrors sampled at 0 , 31, 60, 90 and 124 days post-sterilisation.

\section{DISCUSSION}

There was no recontamination of sterilised instruments in this investigation over the test period of 124 days. This far exceeds the limit of 60 days for pre-wrapped, vacuum sterilised instruments stated by the UK Department of Health. Although the test period is still relatively short, it highlights that the limit of 60 days is not justified if sterilisation has been correctly undertaken. By contrast, within UK hospital central sterile service departments the storage time for sterile instruments is up to 24 months, or until an event occurs that may compromise the integrity of the packaging leading to possible microbial ingress.

This question of sterility being timedependent is not a new one and may have resulted from guidance in 1983 by the Centre for Disease Control in the US, which states that sterilised instruments are only considered to be sterile for one month. ${ }^{14}$ Examination of the subsequent literature has revealed several studies that have investigated the shelf life of sterilised instruments. In one such study Schwarz et $a l .{ }^{15}$ found that only three of 300 sterilised packs became contaminated after 12 months and this was thought to be related to the possibility of inadvertent contamination on opening of the pack, rather than a time-dependent factor. In a separate study of 7,200 packs of sterilised glass rods packaged in either paper envelopes, peel pouches or nylon sleeves, contamination was detected in only a few cases over a 12 month period. This was insignificant in the case of the paper envelopes and peel pouches. For the nylon pouches, 1.5\% demonstrated lack of sterility over 12 months, but again the authors felt this was related to difficulties in opening the packs rather than due to time-related contamination. ${ }^{16}$ Therefore, in both cases the conclusion was that contamination was more likely to be event rather than time-related. In order to test the idea of event-related contamination, Widmer et al. ${ }^{17}$ examined the effects of moving packed instruments over time, as might occur in the course of the normal clinical practice, and found that $8.3 \%$ of instruments were contaminated following such movement. They also concluded that event-related procedures were more likely to affect long-term sterility than time alone. Another study, which examined sterilised medical equipment stored in several hospitals in Australia, found that although several items had sustained an 'event' they still failed to show any contamination of the contained instruments. Interestingly, this study also evaluated the possible cost implications of observing a one-month storage time rule, by auditing those instruments returned for re-sterilisation. It was found that 18,000 items were returned annually as a result. At a conservative estimate of just AUS \$5 per item to cover the manpower and equipments costs, the annual bill to reprocess these unused instruments was estimated to be in the order of AUS $\$ 90,000 .{ }^{18}$

At present there appears to be a lack of evidence in the dental literature on the length of time sterile instruments may remain bagged before use, which brings into question the validity of the current UK guidance to primary care dentists. If a sterilised item can be considered to be sterile almost indefinitely then provided the packaging remains dry and intact, HTM $01-05,{ }^{5}$ with its 60 day recommendation, appears to be placing an extra and possibly unnecessary burden on UK primary care dentists. Such a burden is not without associated costs in materials and manpower as already shown. ${ }^{18}$ At present the guidance on storage times following sterilisation in primary care does not appear to be evidence-based and the recommendations are not supported by this study. 
Authors' contributions: CSB: clinical investigator, study design, data collection and analysis, drafting of the manuscript; VS: expert advice and support relating to microbiological techniques; $D D$ : expert advice relating to microbiological techniques, study design and critical revision; MF: expert advice relating to decontamination and critical revision; JRS: expert advice relating to study design, critica revision; AJI: expert advice relating to study design and final approval of the manuscript, guarantor. All authors who contributed to writing the report have seen and approved the final draft.

The authors state that there are no conflicts of interest. This research forms part of the thesis of the first author for the degree of Doctor of Dental Surgery (DDS) of the University of Bristol.

Funding: this research received a small R\&D research equipment grant from the Royal United Bath NHS Trust, Bath.

1. Smith A J, Hurrell D, Bagg J, McHugh S, Mathewson $H$, Henry M. A method for surveying instrument decontamination procedures in general dental practice. Br Dent J 2007; 202: E20.

2. Bagg J, Smith A J, Hurrell D, McHugh S, Irvine G. Pre-sterilisation cleaning of re-usable instruments in general dental practice. Br Dent J 2007; 202: E22.

3. Smith A J, Bagg J, Hurrell D, McHugh S. Sterilization of re-usable instruments in general dental practice.
Br Dent J 2007; 203: E16.

4. British Dental Association. Infection control in dentistry. Advice sheet A12. London: British Dental Association, 2009

5. Department of Health. Health Technical Memorandum 01-05: decontamination in primary care dental practices. London: Department of Health, 2009.

6. Department of Health. Health Technical Memorandum 2010: sterilisation. London: Department of Health, 1994.

7. Department of Health. Health Technical Memorandum 2030: washer-disinfectors. London: NHS Estates/Department of Health, 1997.

8. Department of Health. Health Technical Memorandum 01-01: decontamination of re-usable medical devices. London: Department of Health, 2007.

9. Department of Health. A guide to the decontamination of reusable surgical instruments. London: NHS Estates/Department of Health, 2003.

10. Medicines and Healthcare Products Regulatory Agency. Sterilization, disinfection and cleaning of medical equipment: guidance on decontamination from the Microbiology Advisory Committee to Department of Health. Part 3 - procedures. London: MHRA, 2006.

11. Rutala W A, Weber D J. Guideline for disinfection and sterilization in healthcare facilities. USA: Center for Disease Control, 2008.

12. Medicines and Healthcare Products Regulatory Agency. Benchtop steam sterilizers webpage. http://www.mhra.gov.uk/Safetyinformation/ Generalsafetyinformationandadvice/ Technicalinformation/Benchtopsteamsterilizers/ index.htm (accessed 20 0ctober 2010).

13. Medical Devices Agency. Benchtop steam sterilizers. Guidance on purchase, operation and maintenance. London: Medical Devices Agency, Department of Health, 2002.

14. Simmons B P. CDC guidelines for the prevention and control of nosocomial infections. Guideline for prevention of intravascular infections. Am J Infect Control 1983; 11: 183-199.

15. Schwartz R, Davis R. Safe storage times for sterile dental packs. Oral Surg Oral Med Oral Pathol 1990; 70: 297-300.

16. Butt W E, Bradley D V Jr, Mayhew R B, Schwartz $R S$. Evaluation of the shelf life of sterile instrument packs. Oral Surg Oral Med Oral Pathol 1991; 72: 650-654.

17. Widmer A F, Houston A, Bollinger E, Wenzel R P. A new standard for sterility testing for autoclaved surgical trays. J Hosp Infect 1992; 21: 253-260.

18. Webster J, Lloyd W, Ho P, Burridge C, George N. Rethinking sterilization practices: evidence for event-related outdating. Infect Control Hosp Epidemiol 2003; 24: 622-624. 